\title{
La Afectividad de la Inteligencia
}

\author{
Luisa M. Morales ${ }^{(1,2)}$ y Orlando E. García ${ }^{(1,2)}$ \\ (1) Universidad de Panamá, Instituto de Estudios Nacionales, Campus Central Octavio Méndez Pereira, \\ Panamá-Panamá (e-mail: lui.mora@hotmail.com). \\ (2) Universidad Especializada de las Américas, Departamento de Ciencias Exactas, Facultad de Salud y \\ Rehabilitación, Paseo Andrews 850, Albrook, Panamá. (e-mail: nangarcia@hotmail.com)
}

Recibido Jun. 25, 2013; Aceptado Jul. 29, 2013; Versión final recibida Ago. 08, 2013

\begin{abstract}
Resumen
Esta investigación analiza la integración de la perspectiva afectiva y cognitiva con los procesos de enseñanza aprendizaje de la matemática. El estudio se llevó a cabo con estudiantes de primer año de universidad. Considerando las características de la muestra el estudio es de tipo descriptivo en el que se caracterizan las actitudes. La matemática es vista usualmente como algo complejo y difícil, lo cual ha originado actitudes desfavorables hacia esta asignatura. Pero tratar que el estudiante analice un problema matemático de manera natural y simple no es tarea sencilla. Eso explica el esfuerzo enorme por parte de los docentes y de las instituciones educativas por intentar modificar la actitud de los estudiantes hacia la matemática. Del estudio se obtuvo correlaciones significativas, mostrando que los alumnos que tienen actitudes positivas hacia el aprendizaje matemático tienen mejores calificaciones también en otras materias y su rendimiento académico general es sobresaliente.
\end{abstract}

Palabras clave: actitud hacia la matemática, rendimiento académico, dominio afectivo, dominio cognitivo

\section{The Affectivity of Intelligence}

\begin{abstract}
This research analyzes the integration of the affective and cognitive perspectives with the teaching and learning process of mathematics. The study was conducted with freshmen university students. Considering the characteristics of the sample the research consist of a descriptive study in which attitudes are characterized. Mathematic is commonly seen as a complex and difficult subject, which have originated unfavorable attitudes to it. This explains the tremendous effort done by teachers and institutions to modify student's attitude towards mathematic. From the study significant correlations were obtained, showing that students who have positive attitudes towards math learning also get better grades in other subjects and have outstanding general academic records.
\end{abstract}

Keywords: attitudes toward mathematics, academic performance, college students, affective domain, cognitive domain 


\section{INTRODUCCIÓN}

Tradicionalmente, las actitudes de los estudiantes hacia la Matemática no han sido siempre las más favorables, gran parte del estudiantado llega a esta asignatura condicionando su punto de vista emocional que se traduce en un rechazo, letargo o apatía. Es evidente que existe una gran problemática en el aprendizaje de la Matemática ya que cada año se refleja un creciente número de fracasos estudiantiles (relacionados con la Matemática), en cualquier parte del mundo. De igual forma se nota que la mayoría de los estudiantes del nivel medio superior adolecen de conocimientos matemáticos básicos, observándose que los estudiantes que egresan de instituciones públicas y en menor medida de escuelas particulares, tienen conocimientos insuficientes en el ámbito del razonamiento matemático.

Por qué será que si se discute de Matemática pareciera que se está hablando de temas extraños que la gente común no son capaces de entender, es decir, como si unos cuantos seres humanos fueron dotados de una especie de traductor en sus cerebros que les permitiera procesar estos conocimientos especializados. Pero qué sucede con los demás, será que piensan que los conocimientos matemáticos nunca los podrán usar, en su entorno social. Esa problemática se pone peor cuando toman una actitud negativa debido a su bajo rendimiento académico en Matemática (Morales, 2009); dejando de manifiesto que la mayoría de estos alumnos (los que tiene una actitud negativa) no ven una utilidad de la Matemática en sus vidas.

La Secretaría de Educación Pública de México (SEP) y el Ministerio de Educación de Panamá (MEDUCA) enfrentan un gran reto: específicamente en relación con la Matemática, mejorar los procesos de aprendizaje para así poder disminuir las dificultades que presentan los alumnos en la comprensión de conceptos, resolución de problemas, transferencia de los contenidos a situaciones cotidianas y, en general, mejorar los procesos y estrategias de pensamiento matemático que les permitan seguir aprendiendo esta ciencia de manera que impacte en la cultura y en la sociedad. Los índices de deserción y reprobación resultan alarmantes, según va evolucionando el estudiante en su educación. Conforme se avanza en los grados educativos, desde el nivel básico hasta el bachillerato, los índices de deserción y reprobación crecen significativamente, disminuyendo la eficiencia terminal notablemente, como se muestra a continuación, en el caso de la educación superior. Los datos reportados varían de acuerdo con la institución y la entidad o institución, por lo que no existe un consenso. La tabla 1 muestra datos conservadores sobre la tasa de deserción y reprobación, según indicadores de la SEP de México y el MEDUCA de Panamá, las fuentes son: *Departamento de estadística del MEDUCA-2012 **Fuente: Instituto Nacional para la Evaluación de la Educación INEE, SEP, México-2011-2012.

Todo lo anterior conlleva al punto que estamos tratando de mostrar, la importancia del desarrollo integral de un individuo en la sociedad capaz de criticar con argumentos lógicos matemáticos ideas erróneas o procesos mal elaborados; lo cual permite la evolución del conocimiento de una ciencia. Esos argumentos lógicos matemáticos solo pueden ser adquiridos mediante el desarrollo de un pensamiento matemático bien estructurado. Políticas educativas internacionales (NTCM Standars) apuntan a la necesidad de que un individuo debe ser capaz de conjeturar como construir contraejemplos donde se muestre la bondad de una información, es decir, que él pueda juzgar o evaluar sus limitaciones como fortalezas. Pero esto indica la importancia de construir un pensamiento matemático, el cual solo puede ser logrado si el sujeto en cuestión imita a un matemático.

Un pensamiento matemático es un pensamiento crítico y científico que se construye de procesos como conceptos matemáticos de forma útil para un individuo dentro de una sociedad (García y Morales, 2012). Este pensamiento no es fácil construir en un ambiente escolar, sino se emplean actividades didácticas que permitan interactuar la información matemática necesaria para la construcción del conocimiento matemático en los alumnos. En ese sentido, es notorio observar los bajos resultados obtenidos por Panamá y México en las pruebas de PISA (Programa que mide las destrezas matemáticas y razonamiento necesarios, en alumnos de 15 años de edad, en el sistema escolar de más de 20 países a nivel internacional). Será que el sistema escolar panameño y mexicano realizan poco esfuerzo, por lo cual, es necesario que las políticas educativas cambien con un objetivo primordial: desarrollar individuos integrales capaces de hacer juicios convenientes y se pueda construir una sociedad que resuelva sus propios problemas. Complementando lo anterior se puede apuntar lo siguiente ¿será que existen pensamientos muy arraigados en los alumnos que no los dejan transitar de un pensamiento ingenuo hacia un pensamiento matemático? Estos pensamientos ingenuos se expresan algunas veces en errores que representan una preocupación en el medio educativo y muchas veces el docente lo caracteriza como un aspecto negativo en el proceso de aprendizaje, pues en su postura, una equivocación de los estudiantes en su trabajo matemático es un fracaso. Algunos autores lo han denominado obstáculo (Brousseau, 1986) en el sentido que impide que se construyan otros conocimientos y surjan nuevas ideas. 
Tabla 1: Indicadores de deserción y reprobación.

\begin{tabular}{|l|c|c|c|c|}
\hline & $\begin{array}{c}\text { Tasa de deserción } \\
\text { Panamá* }^{*}\end{array}$ & $\begin{array}{c}\text { Tasa de reprobación } \\
\text { Panamá* }\end{array}$ & $\begin{array}{c}\text { Tasa de deserción } \\
\text { México }^{* *}\end{array}$ & $\begin{array}{c}\text { Tasa de reprobación } \\
\text { México }^{* * *}\end{array}$ \\
\hline Primaria & 0.6 & 5.7 & 0.6 & 2.1 \\
\hline Secundaria & 10.6 & 6.3 & 5.3 & 14.5 \\
\hline Preparatoria & 12.7 & 2.3 & 14.5 & 11.5 \\
\hline
\end{tabular}

Una parte de la problemática escolar no radica en la capacidad o incapacidad de los alumnos para comprender determinados temas, sino en su actitud hacia la escuela, el profesor y la asignatura. Autores proponen que el éxito de un alumno en clase de Matemática, está en relación con la actitud positiva hacia la actividad que realice de esta materia y que se incluyen rasgos de personalidad que involucran las esferas intelectual y emocional. Esto conduce a la observación de que las actitudes en la educación básica están en dependencia con las creencias que se reciben desde la infancia siendo afirmadas por el impacto verbal y el comportamiento de la familia, en la vida en comunidad y en la escuela. Distintos estudios (Guerrero y Blanco, 2002; Akey, 2006; Morales, 2009) han reportado que las actitudes muestran una correlación positiva muy significativa con la motivación, la disciplina, el aprendizaje y la conducta en general. Por ello, resulta de suma importancia profundizar en su formación. Entre las personas se pueden encontrar distintas percepciones o concepciones sobre la Matemática que lo conducen a mostrar ciertas actitudes hacia ella; y "distintas investigaciones han puesto de manifiesto que el éxito y fracaso en Matemática depende de algo más que del conocimiento de ciertos requisitos de contenido matemático. Conocer apropiadamente hechos, algoritmos y procedimientos no es suficiente para garantizar el éxito" (Gómez-Chacón, 2009).

Encontrar una respuesta a la pregunta ¿existe una relación entre lo afectivo y cognitivo? es el motivo principal que ha llevado a realizar la presente investigación. Los autores de este trabajo consideran que en la medida en que se investiguen aspectos de naturaleza psicológica en el proceso educativo, en este caso la actitud, se obtendrá información que permitirá hacer propuestas orientadas a mejorar el proceso de enseñanza-aprendizaje. De esta manera el objetivo del trabajo es analizar y comparar la relación existente entre la actitud hacia la Matemática y el rendimiento escolar en estudiantes universitarios de Panamá y México.

\section{METODOLOGÍA}

Debido a las características de la muestra y al problema de la investigación, se trata de un estudio descriptivo -en el que se caracterizan las actitudes- y correlacional -con el propósito de medir el grado de relación que existe entre las variables del estudio- (Hernández, Fernández y Baptista, 2010). Su alcance temporal, corresponde a un estudio transversal, pues es observada en un solo punto en el tiempo. Su amplitud según Sierra Bravo (1992), es microsociológica, pues se estudiaron los fenómenos que se desean investigar en grupos reducidos, sin pretensión de generalizar los resultados de los mismos, cuyo esquema es el siguiente:

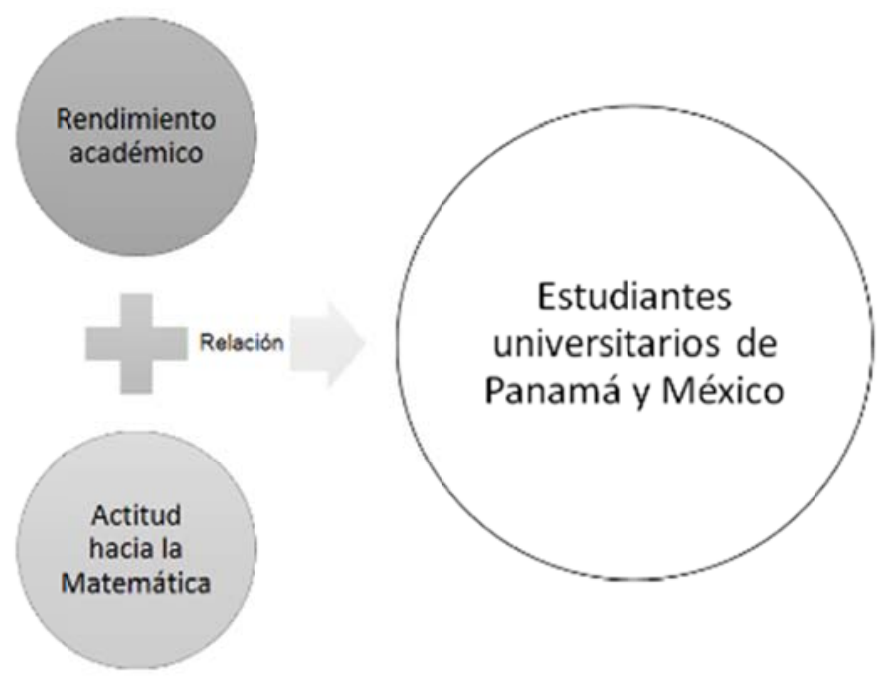

Fig. 1: Esquema sobre la metodología de la investigación

El estudio se desarrolló en dos fases para la encuesta: en la primera, se diseñó un instrumento de escala de actitudes y se aplicó a los estudiantes de la Universidad Autónoma del Estado de Hidalgo (UAEH) y de la Universidad de Panamá (UP); este cohorte fue de un total de 247 alumnos, de los cuales 120 son de la 
Facultad de Ciencias Naturales, Exactas y Tecnología (UP) y 127 del Instituto de Ciencias Básicas e Ingeniería (UAEH), para su validación. En la segunda fase se llevó a cabo la aplicación a los estudiantes de primer ingreso del 2012 de las mismas universidades con una muestra total de 1076 estudiantes, donde 530 son de Panamá y 546 son de México para el estudio correspondiente correlacional de variables.

La primera fase del estudio tiene un carácter exploratorio, ya que se trató de lograr un acercamiento a las actitudes de los estudiantes encuestados de licenciaturas e ingenierías de carácter científico para la validación del instrumento, sin tomar en cuenta el semestre que cursara. Mientras que, en la segunda fase se tomaron los grupos en donde el total de estudiantes eran de nuevo ingreso, y se eliminó de la población, los alumnos reprobados en la materia evaluada (Matemática); ya que estos grupos podrían representar un sesgo en la información, afectando a las variables estudiadas.

La selección de la segunda fase se determinó mediante un muestreo estratificado, el cual se utiliza cuando la población se encuentra dividida de manera natural en grupos que contienen la variabilidad de la población. Los estratos representados por las universidades participantes se exponen en las tablas 1 y 2 . En esta se presenta por universidad el número de estudiantes matriculados por carrera en el año 2012, así como el tamaño de la población y tamaño de muestra estimado. Para la determinación del tamaño de muestra se estableció un nivel de confianza del $95 \%$ y un error de estimación del $5 \%$. La población está compuesta por estudiantes universitarios de primer ingreso, de ambos sexos, de México del ciclo escolar 2011-2012 del ICBI de la UAEH, y de Panamá de la UP, en las siguientes facultades: Facultad de Ciencias Naturales, Exactas y Tecnología; Facultad de Arquitectura; Facultad de Electrónica y Telecomunicaciones.

Tabla 2: Población y muestra de estudiantes, generación 2011-2012

\begin{tabular}{|c|c|c|}
\hline Universidad Autónoma del Estado de Hidalgo & Población & Muestra \\
\hline Licenciatura Ingeniería Industrial & 110 & 86 \\
\hline Licenciatura Ingeniería Civil & 85 & 70 \\
\hline Licenciatura Biología & 45 & 41 \\
\hline Licenciatura Ingeniería en Geología Ambiental & 34 & 27 \\
\hline Licenciatura en Química & 37 & 34 \\
\hline Licenciatura Química en Alimentos & 45 & 41 \\
\hline Licenciatura Sistemas Computacionales & 137 & 102 \\
\hline Licenciatura Arquitectura & 116 & 84 \\
\hline Licenciatura Ingeniería en Electrónica y Telecomunicaciones & 66 & 57 \\
\hline Licenciatura en Ingeniería en Ciencias de los Materiales & 4 & 4 \\
\hline $\begin{array}{ll} & \text { Total en México } \\
\end{array}$ & 679 & 546 \\
\hline
\end{tabular}

\section{Rendimiento académico y sus indicadores}

En este trabajo el rendimiento académico se define operacionalmente con la media de las calificaciones obtenidas en la asignatura al finalizar el curso enero-julio 2012 en México, y marzo-julio 2012 en Panamá.

Tabla 3. Estratos: población y muestra de estudiantes -Generación 2012

\begin{tabular}{|c|c|c|}
\hline Universidad de Panamá & Población & Muestra \\
\hline Licenciatura en Física & 7 & 7 \\
\hline Licenciatura en Matemática & 22 & 22 \\
\hline Licenciatura Biología & 206 & 136 \\
\hline Licenciatura Ingeniería Geológica & 12 & 12 \\
\hline Licenciatura en Química & 49 & 49 \\
\hline Licenciatura en Ingeniería Química Industrial & 24 & 24 \\
\hline Licenciatura en Informática & 116 & 90 \\
\hline Licenciatura Arquitectura & 287 & 165 \\
\hline Licenciatura Ingeniería en Electrónica y Telecomunicaciones & 22 & 21 \\
\hline Licenciatura en Topografía y Geodesia & 4 & 4 \\
\hline Total en Panamá & 749 & 530 \\
\hline
\end{tabular}

Para fines prácticos y de acuerdo con la hipótesis, se han definido cuatro categorías diferentes para esta variable, pero la principal para este estudio es el rendimiento académico sobresaliente, pues se considera al estudiante de alto mérito individual; es decir, se observa una alta congruencia entre lo que se le enseña y lo que él demuestra poseer al término del proceso educativo, la condición de éxito y fracaso escolar se centra en el alumno. 
Rendimiento insuficiente: corresponde a una calificación de 0 para el estudiante de la UAEH y 0 a 60 para el estudiante de la UP.

Rendimiento deficiente: se consideran dentro de este grupo todos los estudiantes que tengan una calificación entre 1 a 5 de la UAEH y 61 a 70 para el estudiante de la UP.

Rendimiento regular: en este grupo están englobados los alumnos que tienen una calificación de 6 a 7 en la UAEH y 71 a 80 los estudiantes de la UP.

Rendimiento Sobresaliente: se encuentran aquí los estudiantes que tienen una puntuación dentro del recorrido de calificación de 8 a 10 en la UAEH y 81 a 100 en la UP.

En el sistema de evaluación del aprendizaje escolar que rige a la Universidad Autónoma del Estado de Hidalgo (UAEH), la mayor parte de las calificaciones se basan en el sistema decimal, es decir de 0 a 10. En la Universidad de Panamá (UP) la calificación se asigna en una escala de 0 a 100, basado en letras. Estos dos sistemas en los cuales el puntaje obtenido se traduce en la categorización del logro de aprendizaje pueden variar desde aprendizaje bien logrado hasta aprendizaje deficiente. En este estudio se adoptaron los criterios empleados en los sistemas de evaluación de ambas instituciones educativas para conceptualizar el rendimiento académico en Matemática (tabla 4). En México y Panamá el rendimiento académico es el proceso alcanzado por los alumnos en función de los objetivos programados.

Tabla 4: Categorización del nivel de rendimiento académico

\begin{tabular}{|c|c|}
\hline \multicolumn{2}{|c|}{ México } \\
\hline Escala & Calificación \\
\hline 0 & Insuficiente (I) \\
\hline 1 a 5 & Deficiente (D) \\
\hline 6 a 7 & Regular (R) \\
\hline 8 a 9 & Bueno (B) \\
\hline 10 & Excelente (E) \\
\hline
\end{tabular}

Sobresaliente

\begin{tabular}{|c|c|}
\hline \multicolumn{2}{|c|}{ Panamá } \\
\hline Escala & Calificación \\
\hline 0 a 60 (F) & Insuficiente \\
\hline 61 a $70(\mathrm{D})$ & Deficiente \\
\hline 71 a $80(\mathrm{C})$ & Regular \\
\hline 81 a $90(\mathrm{~B})$ & Bueno \\
\hline 91 a $100(\mathrm{~A})$ & Excelente \\
\hline
\end{tabular}

\section{Actitud y sus indicadores}

La actitud se trata como una variable de tipo cuantitativa que se relaciona con sus componentes cognitivo, afectivo y conductual. La actitud hacia la Matemática se define, según Petriz, Barona, López y Quiroz (2010), como una serie de disposiciones que manifiesta el individuo para aceptar familiarizarse o no, con determinados contenidos matemáticos.

Esta variable es tratada como categórica; es una escala calificada en un nivel positivo y negativo, en cuanto a los componentes, de cognitiva, afectiva y conductual, por medio del baremo elaborado por Morales (2009), adaptada a los dos países. Cada componente posee indicadores, que son los puntajes obtenidos por la muestra en la Escala de actitudes. Entonces la medición de esta variable se realizó considerando varias categorías para cada uno de los componentes de la actitud entre una muestra de estudiantes durante el semestre enero-julio 2012. En la tabla 5 se presentan las categorías que conformaron cada uno de los componentes en la escala de actitud hacia la Matemática creada para este estudio.

Tabla 5: Componente e indicadores dentro de la escala de actitud hacia la Matemática

\begin{tabular}{|c|c|}
\hline $\begin{array}{ll}\text { Indicadores } & \text { Componente Cognitivo }\end{array}$ & $\begin{array}{l}\text {-.Competencia Matemática. } \\
\text {-. Ansiedad }\end{array}$ \\
\hline $\begin{aligned} & \text { Indicadores } \\
& \text {-. Motivación. } \\
& \text {-. Bloqueo Emocional. }\end{aligned}$ & $\begin{array}{l}\text {-. Interés. } \\
\text {-. Aceptación. }\end{array}$ \\
\hline $\begin{array}{l}\text { Indicadores } \\
\text {-. Rechazo. } \\
\text {-. Compañerismo. }\end{array}$ & $\begin{array}{l}\text { Ial } \\
\text {-. Confianza. } \\
\text {-. Curiosidad. }\end{array}$ \\
\hline
\end{tabular}

\section{RESULTADOS}

Se presenta en primer lugar un análisis descriptivo realizado para caracterizar la muestra según las diferentes variables incluidas en el estudio. Se calcularon medidas de tendencia central y de dispersión. Los resultados se presentan en tablas y figuras. 


\section{Análisis del rendimiento académico en Matemática de los estudiantes participantes}

En la figura 2 se presenta el nivel de rendimiento académico logrado por los estudiantes de la muestra de ambos países. Como se indicó anteriormente, se tomó en cuenta la conceptualización sobre rendimiento propuesta por Lent, Brown y Hackett. Es así que los estudios enfocados a las problemáticas existentes en el proceso de enseñanza-aprendizaje de la Matemática han incorporado variables de tipo afectivo y socioculturales, los cuales han concluido que el factor cognitivo no es el único participante en el aprendizaje, ya que es un proceso compartido entre el ámbito afectivo y el contexto del sujeto que aprende (Planchart, Garbín y Gómez-Chacón, 2005).

\section{Descripción de las actitudes en la muestra total}

Los datos recopilados con la escala de actitud hacia la Matemática se analizaron inicialmente empleando un análisis de distribución de frecuencias organizado de dos formas: con la muestra total y disgregándolos por país. Se encontró que los estudiantes muestran una concentración en los puntos 3 y 4 de la escala, lo cual implica que tienen una actitud de indecisión a parcialmente positiva. Además hay una escasa frecuencia en los puntos 2 (actitud negativa) y 5 (actitud positiva) de la escala. Si bien tienden a ser favorables en un principio, la evolución negativa que se produce a lo largo del tiempo y la persistencia de este matiz desfavorable son características muy específicas que conviene tener presentes para poder entender reacciones futuras del alumno e intervenir adecuadamente en ellas (Auzmendi, 1992).

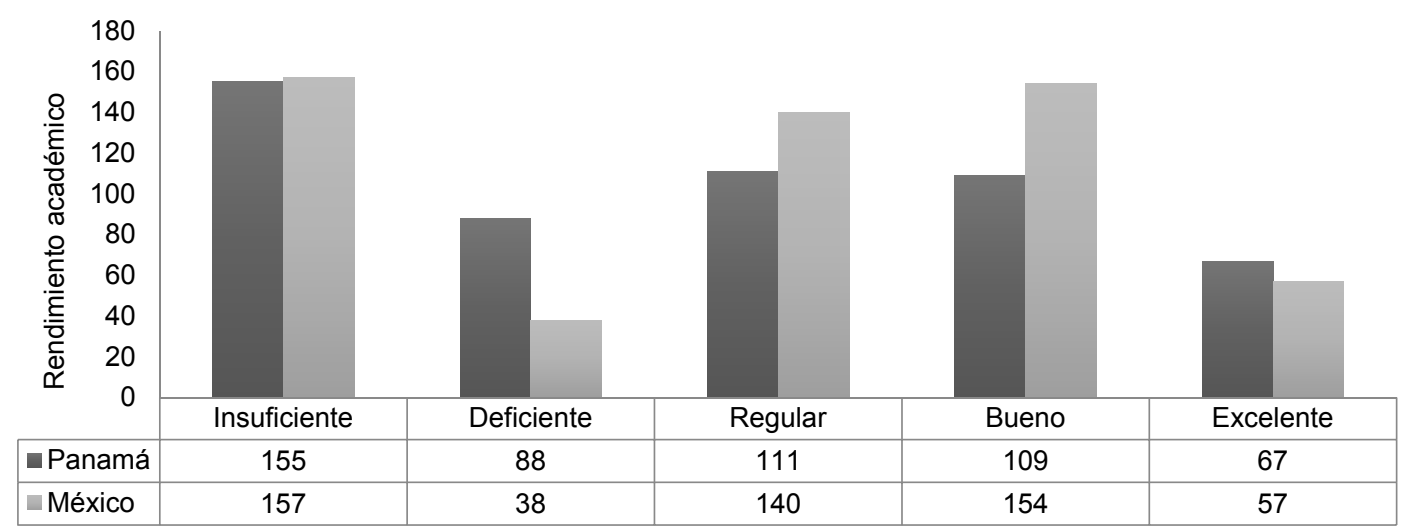

Fig. 2: Distribución de frecuencia del rendimiento en Matemática en los estudiantes de la muestra

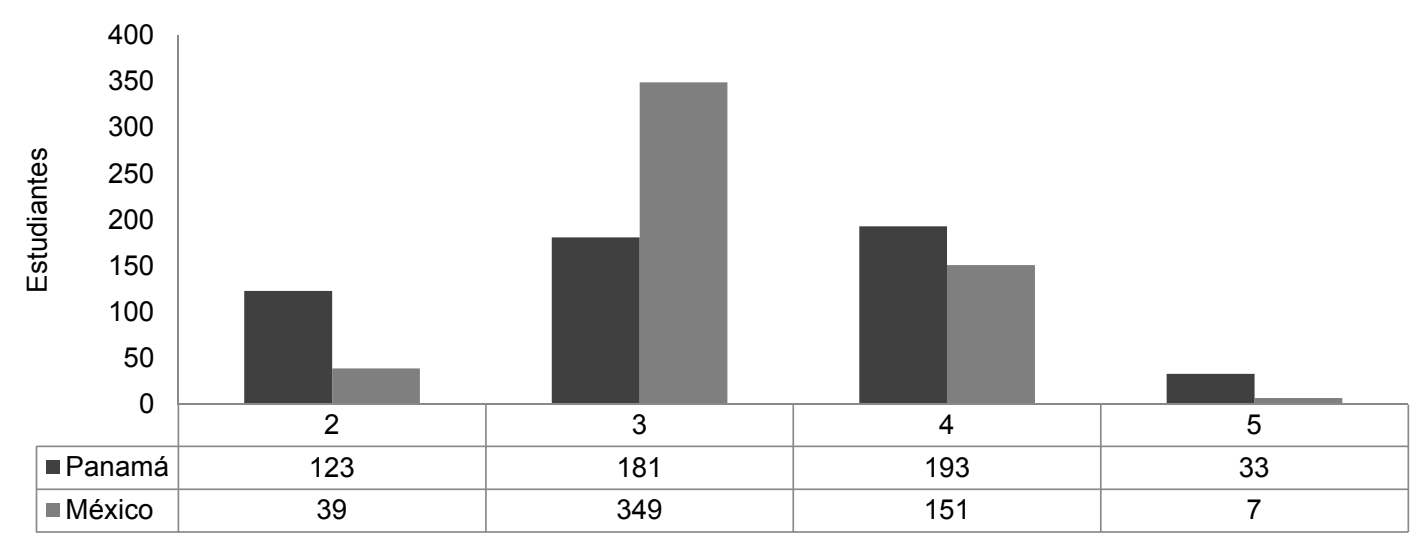

Fig. 3: Distribución de frecuencias en los puntos de la escala de actitud hacia la Matemática.

\section{Correlación entre el rendimiento académico y la actitud}

Otro tipo de análisis de los datos obtenidos sobre las actitudes hacia las Matemática consistió en evaluar la relación entre estas y el rendimiento académico en Matemática. Para esto se empleó el coeficiente de correlación de Spearman. Se correlacionaron las puntuaciones obtenidas en la Escala de Actitud con las calificaciones de los estudiantes en el periodo escolar enero-julio 2012. En la tabla 6: ${ }^{* *}$ Significancia al nivel de 0,$01 ;$ * Significancia al nivel de 0,05 . 
Tabla 6: Correlación de las variables en estudio

\begin{tabular}{|c|c|c|c|c|}
\hline \multicolumn{2}{|c|}{} & \multicolumn{3}{|c|}{ Rendimiento académico } \\
\hline \multirow{2}{*}{ Actitud } & General & Panamá & México \\
\cline { 2 - 5 } & Coefic. $(\rho)$ & $0.788^{* *}$ & $0.823^{\star *}$ & $0.745^{\star}$ \\
\cline { 2 - 5 } & Sig. & .000 & .000 & .000 \\
\hline
\end{tabular}

\section{Correlaciones}

Los resultados del análisis sugieren que el rendimiento académico correlaciona positivamente con la actitud. El valor del coeficiente de correlación osciló entre $.745(\rho \leq .05)$, en los estudiantes de México y $0.823(\rho<$ 0.01 ), en los estudiantes de Panamá. Es decir, la actitud positiva hacia la Matemática está relacionada con un mayor rendimiento académico en la materia.

De este modo, los resultados indican que en los estudiantes de primer ingreso de ambas universidades las actitudes hacia la Matemática están asociadas con su rendimiento académico en Matemática: en particular, hay una propensión a que un mayor rendimiento en la materia se corresponde con una actitud hacia las Matemática más positiva (figura 4). La implicación de esto, en concordancia con Auzmendi (1992), es que: "las actitudes influyen en el proceso de aprendizaje y, a su vez, la educación tiene un amplio poder sobre ellas. Así, se pretende mejor aquello que concuerda o es congruente con nuestras propias actitudes o lo que produce mayor agrado, y una educación adecuada puede mejorarlas en los estudiantes ante un área determinada" (p. 18).

Estos aspectos también fueron estudiados recientemente por Lim, Tso y Lin (2009), quienes concluyeron que las actitudes hacia esta disciplina son un fenómeno multidimensional; encontraron que estos componentes influyen en el aprendizaje de los alumnos en el sentido de brindarlles un panorama de las conexiones de las Matemática con el mundo real. Los hallazgos de esta investigación son similares a los encontrados por Álvarez y Soler (2010) en lo que respecta a que los alumnos perciben a la Matemática como una disciplina útil, no sólo en el ámbito académico sino también laboral. Sin embargo, manifiestan desconfianza y ansiedad en las situaciones que involucran el empleo de los procedimientos matemáticos.
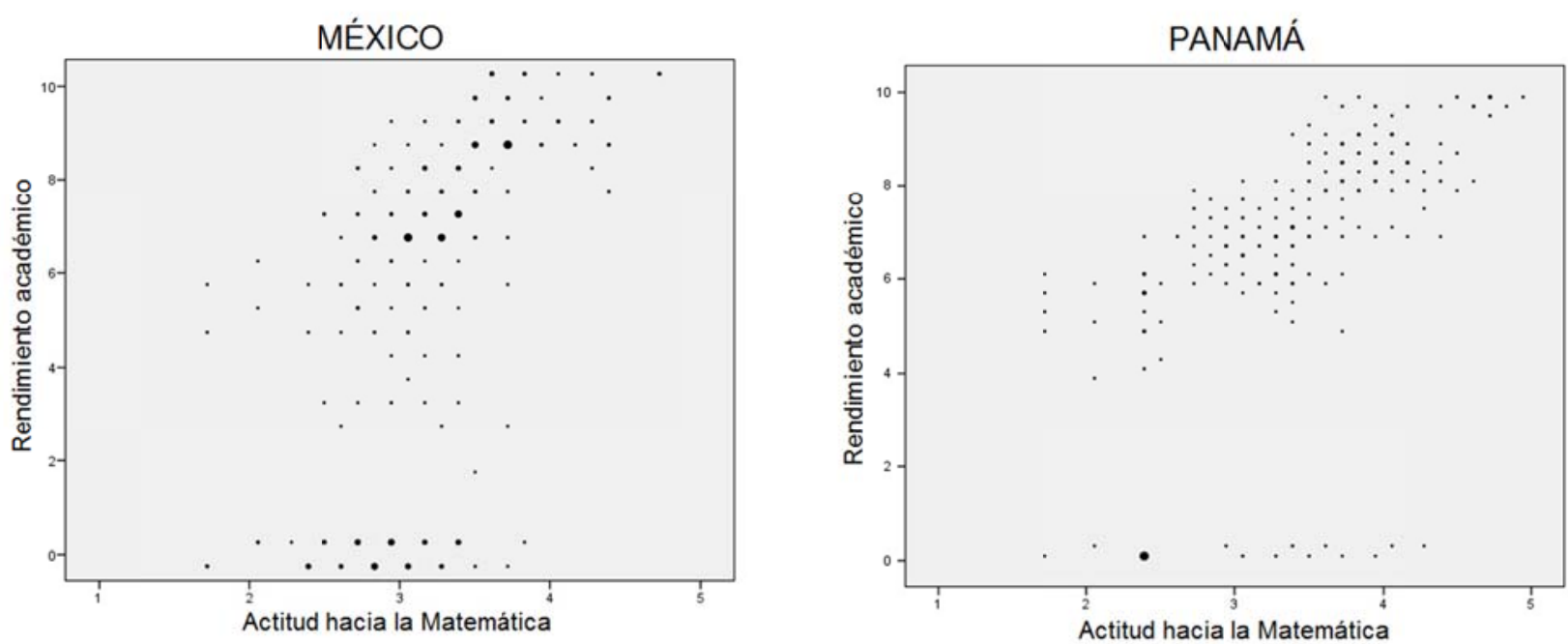

Fig. 4: Diagrama de dispersión rendimiento académico y actitud en Matemática.

En la figura anterior se aprecia que el valor obtenido da como resultado una correlación fuerte entre actitud y rendimiento académico en Panamá y México, siendo la estimación de este valor el que está directamente vinculado con objetivo planteado en esta investigación. El resultado obtenido sugiere que a mayor actitud hacia la Matemática del estudiante mayor rendimiento académico. Adicionalmente, se evaluó la correlación existente entre el rendimiento académico y cada una de las componentes de la actitud: la cognitiva, la afectiva y la conductual. Los resultados obtenidos se muestran en la tabla 7 , como se puede apreciar en los resultados, cada una de las dimensiones medidas por el instrumento utilizado muestra una relación significativa con el rendimiento académico. En un orden decreciente, en cuanto a su grado de correlación, se ubicarían como sigue: cognitiva $(r=0.771, \rho=0)$, conductual $(r=0.751, \rho=0)$ y afectiva $(r=0.591, \rho=0)$ con una $^{* *}$ significancia al nivel de 0,01 (bilateral). 
Tabla 7: Correlación del Rendimiento Académico con cada una de los Componentes de la Actitud.

\begin{tabular}{|l|c|c|c|c|c|c|c|}
\hline & & \multicolumn{2}{|c|}{ Dimensión Cognitiva } & \multicolumn{2}{c|}{ Dimensión Afectiva } & \multicolumn{2}{c|}{$\begin{array}{l}\text { Dimensión } \\
\text { Conductual }\end{array}$} \\
\hline & & Panamá & México & Panamá & México & Panamá & México \\
\hline $\begin{array}{l}\text { Rendimiento } \\
\text { Académico }\end{array}$ & Coefic. $(\rho)$ & $.637\left(^{* *}\right)$ & $.466\left(^{* *}\right)$ & $.749\left(^{* *}\right)$ & $.573\left(^{* *}\right)$ & $.749\left(^{* *}\right)$ & $.641\left(^{* *}\right)$ \\
\hline & Sig. & .000 & .000 & .000 & .000 & .000 & .000 \\
\hline $\begin{array}{l}\text { Dimensión } \\
\text { Cognitiva }\end{array}$ & Coefic. $(\rho)$ & & $.587\left(^{* *}\right)$ & $.285\left(^{* *}\right)$ & $.660\left(^{* *}\right)$ & $.354\left(^{* *}\right)$ \\
\hline & Sig. & & .000 & .000 & .000 & .000 \\
\hline $\begin{array}{l}\text { Dimensión } \\
\text { Afectiva }\end{array}$ & Coefic. $(\rho)$ & & & & $.803\left(^{* *}\right)$ & $.520\left(^{* *}\right)$ \\
\hline & Sig. & & & & & & \\
\hline
\end{tabular}

En la tabla 7 se observa que existe una correlación positiva significativa entre el rendimiento académico y la dimensión cognitiva en Panamá $(r=.637, \rho=0)$ - México $(r=.466, \rho=0)$, lo que indica que existe una alta probabilidad que el desarrollo cognitivo del estudiante influya directamente en el rendimiento académico de manera positiva, lo que implica que a mayor comprensión de los conocimientos matemáticos mejor será su rendimiento académico. En la tabla 6 se puede observar que el valor obtenido da como resultado una fuerte correlación entre actitud y rendimiento académico en Panamá y México, siendo la estimación de este valor el que está directamente vinculado con objetivo planteado en esta investigación. Al parecer el resultado obtenido corresponde con la expectativa del estudio: a mayor actitud hacia la Matemática del estudiante será mayor su rendimiento académico.

Por otro lado, en la figura 5 se observa que el desarrollo cognitivo de un individuo está íntimamente ligado en la Matemática con la resolución de problemas con la relación de las preguntas 14 (Resolver un problema matemático es saber analizar y aplicar los conocimientos) y 28 (En clase de Matemática me siento ansioso al resolver un problema). Sin embargo, la mayoría de los encuestados están conscientes que resolver un problema implica un análisis exhaustivo, la aplicación de conceptos y una estrategia bien marcada como sostiene Polya (2001). Se puede observar que la mitad de los estudiantes consideran que presentan sentimientos de ansiedad que influye en la resolución de problemas matemáticos y se manifiesta en rendimientos académicos deficientes. Con esta correlación encontrada se puede establecer una interdependencia entre el desarrollo cognitivo de un sujeto y su actitud frente a resoluciones de problemas específicamente en el caso de la Matemática.

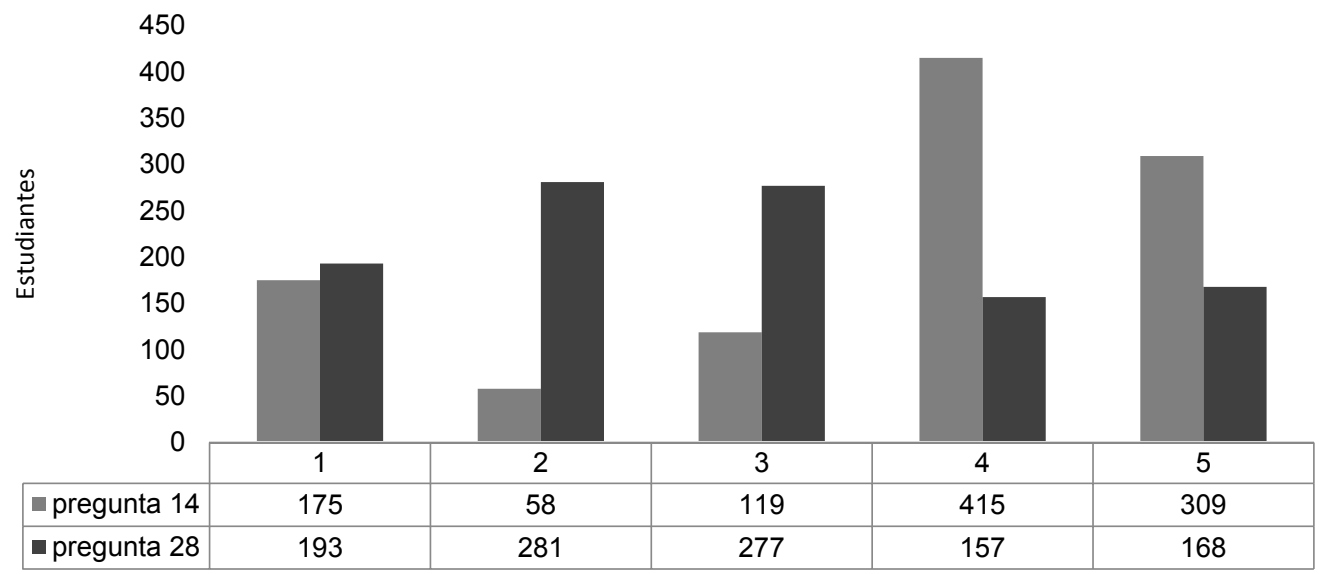

Fig. 5: Comparación de elementos cognitivos y afectivos

Tomando en cuenta lo expuesto hasta aquí, se puede considerar positivo el incorporar de nuevo en el tratamiento curricular de la Matemática los enfoques empirocéntricos y psicocéntricos. El primero exige hacer una Matemática más ligadas a la experiencia e implica la aplicación de los principios de realidad, necesidad y utilidad. El segundo, supone respetar las características de la persona que aprende y conlleva la realización de objetivos y actividades de acuerdo a sus posibilidades, los estados de ánimo positivos causan que las gente piense, sienta y actue en formas que promueven tanto la construcción de recursos como la generación de vínculos (Ramírez y Fuentes, 2013). 
La consideración de los enfoques citados, así como la utilización de adecuadas estructuras organizativas (por ejemplo, agrupaciones flexibles de alumnos), ha de evitar los enormes desfases que se dan en la actualidad entre las propuestas didácticas y las posibilidades de aprendizajes de los alumnos, es por ello viable que los enfoques empirocéntricos y psicocéntricos, se integren a nivel de la planificación curricular, lo que supone una modificación de los programas de aprendizaje y una adecuación más coherente a las posibilidades cognitivas de nuestros estudiantes (Gairín, 1987, p.138). Lo anterior supone, por ejemplo y entre otras cosas, el abandonar la idea de que para cada curso escolar debe existir un aumento de los conocimientos matemáticos. Farías y Pérez (2010), manifiestan que para conseguir que los alumnos aprendan, no basta explicar bien la materia y exigirles que aprendan. Es necesario despertar su atención, crear en ellos un genuino interés por el estudio, estimular su deseo de conseguir los resultados previstos y cultivar el gusto por los trabajos escolares.

\section{CONCLUSIONES}

Se han obtenido elementos que apoyan la hipótesis inicialmente formulada para este trabajo, dado que se alcanzaron correlaciones significativas entre las actitudes hacia la Matemática y el rendimiento académico por parte de los estudiantes universitarios, ya que los alumnos que tienen actitudes positivas hacia el aprendizaje matemático tienen mejores calificaciones y su rendimiento académico es sobresaliente. Dentro de los aspectos encontrados se presentan fuertes vínculos entre el desarrollo de resoluciones de problemas matemáticos (componente cognitivo) y la parte afectiva de un sujeto. Se recomienda, a su ingreso a la universidad al estudiante deba hacer una serie de ajustes para adaptarse a las exigencias de este nivel educativo. Con frecuencia estos alumnos provienen de un nivel medio superior en donde ha desarrollado hábitos de estudios deficientes o inadecuados e incluso pueden tener alguna deficiencia en habilidades.

Para ambos sistemas educativos resultan relevantes los estudios en torno a esta temática, debido a las carencias de una gran cantidad de alumnos en torno a competencias matemáticas básicas para un desempeño adecuado en distintos ámbitos de la vida.

A partir de este trabajo, se sugiere propiciar el desarrollo de buenas actitudes hacia el aprendizaje matemático de los estudiantes, por medio de acciones educativas y motivacionales por parte de los profesores, e incluso de todo el sistema educativo. Esto es, el docente es uno de los actores principales del fenómeno educativo, ya que no sólo transmite contenidos, sino que también fomenta valores, creencias, necesidades y, por supuesto, actitudes.

\section{AGRADECIMIENTOS}

Investigación financiada por la Secretaria de Ciencia y Tecnología (SENACYT) en el programa Nuevos Investigadores 2011.

\section{REFERENCIAS}

Akey, T. School context, student attitudes and behavior, and academic achievement: an exploratory analysis (en línea), 2006, http://www.mdrc.org/sites/default/files/full_519.pdf. New York: MDRC. Acceso: 18 de noviembre (2012).

Álvarez, Y. y Soler, M. Actitudes hacia las matemáticas en estudiantes de ingeniería en universidades autónomas venezolanas. Venezuela: Revista de Pedagogía, 31(89), 225 - 249, (2010).

Auzmendi, E. Las actitudes hacia la Matemática estadística en las enseñanzas medias y universitarias. Mensajero, Bilbao, España (1992).

Brousseau G. Fundamentos y métodos de la Didáctica de la Matemática, Universidad Nacional de Córdoba, Facultad de Matemática Astronomía y Física, Serie B, Trabajos de Matemática, No. 19 (versión castellana 1993).

García, O. y Morales, L., La incorporación de la conjetura y el contraejemplo: En el aprendizaje de la Matemática. Editorial Académica Española, 3-4, Saarbrücken, Alemania (2012).

Gómez-Chacón, I., Actitudes Matematices: propuestas para la transición del bachillerato a la universidad. Educación Matemática, 21, 3, 5-32. (2009).

Guerrero, E. y Blanco L. J. Estudio preliminar sobre la influencia de las emociones y los estilos cognitivos ante la tarea académica. En Fajado, M. I. y otros. Psicología de la educación y formación del profesorado. Nuevos retos, nuevas respuestas. INFAD, (2002). 
Gairín, J., Las actitudes en educación. Un estudio sobre Matemáticas. 138, Promociones y Publicaciones Universitarias, Barcelona, España (1987).

Hernández, R., Fernández, C. y Baptista, P. Metodología de la investigación (quinta edición). McGraw-Hill, México (2010).

INEE Panorama Educativo de México. Indicadores del Sistema Educativo Nacional. (en línea). 2010. http://educacionadebate.org/wp-content/uploads/2012/09/INEE.Panorama-2011.pdf. Acceso 02 de abril (2013).

Lent, R., Brown, D y Hackett,G. Toward a Unifying Social Cognitive Theory of Career and Academic Interest, Choice, and Performance. Journal of Vocational Behavior 45, 79-122, (1994).

Lim, L., Tso, T. y Lin, F. Assessing science student's attitudes to mathematics: a case study on a modeling project with mathematical software. Int. J. of Mathematical Education in Science and Technology, 40, 4, 441-453, (2009).

MEDUCA Dirección Nacional de Planeamiento Educativo 2010 . (en línea). http://www.meduca.gob.pa/Estadistica_Educativa/2010/INDICADORES_2010_(PRELIMINAR).pdf.

Acceso 02 de abril (2013)

Morales, L. Influencia de la actitud en el rendimiento académico en matemática con estudiantes universitarios. Tesis de maestría no publicada, Universidad Autónoma del Estado de Hidalgo, México, (2009).

Polya, G. Cómo plantear y resolver problemas. (J. Zagazagoitía, Trad). Trillas, México (2001).

Petriz, M. A., Barona C., López, R. M. y Quiroz, J. Niveles de desempeño y actitudes hacia las Matemáticas en estudiantes de la Licenciatura en Administración en una universidad estatal mexicana. Revista Mexicana de Investigación Educativa, 15(47), 1223-1249, (2010).

Planchart, E., Garbín, S. y Gómez-Chacón, I. Enseñanza de la matemática en Venezuela. En GómezRodríguez, A. Psicología Social. Trillas, México (1991).

Ramírez, P. y Fuentes, C. Felicidad y Rendimiento Académico: Efecto Moderador de la Felicidad sobre Indicadores de Selección y Rendimiento Académico de Alumnos de Ingeniería Comercial. Formación Universitaria. 6(3) 21-30 (2013).

SEP. indiSEP (Sistema de Indicadores de la Secretaría de Educación Pública.) [En línea]. www.sep.gob.mx. Acceso 02 de abril (2012).

Sierra Bravo, R. Técnicas de investigación social. Teorías y ejercicios. Paraninfo, Madrid, España (1992)

Farías, D. y Pérez, J. Motivación en la Enseñanza de las Matemáticas y la Administración. Formación universitaria, 3(6), 33-40 (2010). 